\title{
Human Activity Recognition: Literature Review
}

\author{
Maisirreem A. Kamal', Dena Rafaa Ahmed ${ }^{2}$, Rashad Adhed Kamal ${ }^{3}$ \\ ${ }^{1 *, 2}$ College of Computer Sciences and Mathematics, University of Mosul, Mosul, IRAQ \\ ${ }^{3}$ College of Engineering, University of Mosul, Mosul, IRAQ
}

E-mail: ${ }^{1 *}$ maisirreem_alsaigh@uomosul.edu.iq, ${ }^{2}$ dinasalimagha@uomosul.edu.iq, ${ }^{3}$ rashad.alsaigh@gmail.com

(Received May 25, 2021; Accepted June 29, 2021; Available online December 01, 2021)

DOI: 10.33899/edusj.2021.130293.1162, (C) 2021, College of Education for Pure Science, University of Mosul.

This is an open access article under the CC BY 4.0 license (http://creativecommons.org/licenses/by/4.0/).

\begin{abstract}
:
Human activity recognition has an important role in the interaction between human and human relationships because it provides information about a person's identity, personality, activities, psychological state, and health, all this information is difficult to extract due to the difficulty of a person's ability to identify the activities of another person and is considered one of the basic research topics in the scientific fields in the field of computer vision and machine learning. the purpose of human activity recognition (HAR) is to identify the different human activities throw monitoring and register the human activates and the various surrounded environment, by using computers, the human activity recognition researches which depending on visions is the basics of lots of applications even video monitoring or health care and security monitoring and the interaction between the human and the computers.

In this research, a review of the newest development in the human activity recognition branch have been studied, and the different ways to recognize the human actions, an important detail have been shown to preview the HAR researches and the methodologies used to represent the human activates and its classifications, to provide an overview of the HAR methods and comparing them.
\end{abstract}

Keywords: Human Activity Recognition( HAR), machine learning algorithm, smart phone sensors, wearable sensor device.

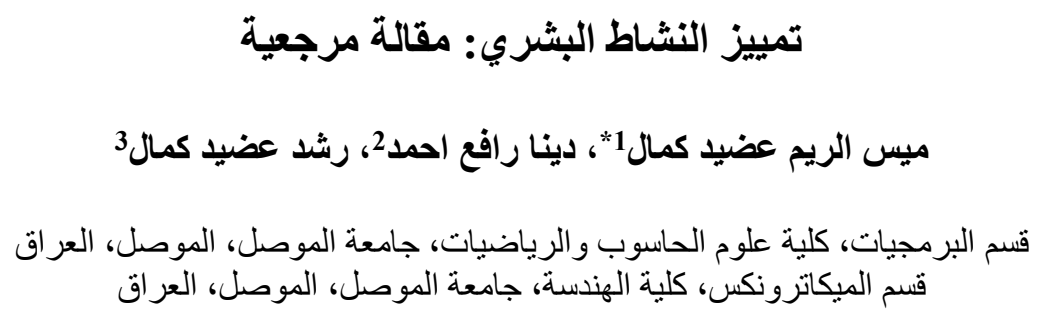

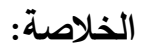

يلعب التعرف على النثاط البشري دورًا مهمًا في التفاعل بين العلاقات الإنسانية والإنسانية لأنه يوفر معلومات حول هوية الثخص

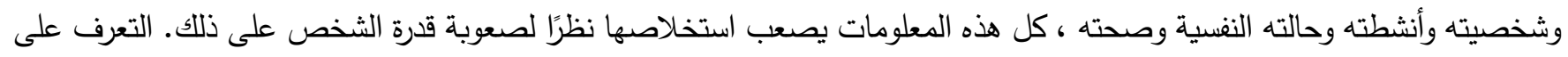
أنشطة شخص آخر وتعتبر من موضوعات البحث الأساسية في الهجالات العلمية في مجال الرؤية الحاسوبية والتعلم الآلي. 
يعدف التعرف على النشاط البشري (Human Activities Recognition( HAR) ) إلى التعرف على الأنشطة البشريه من خلال مراقبه وتسجيل فعاليات الأشخاص والظروف البيئية المحيطه بهم باستخدام الحاسوب، حيث ان أبحاث التعرف على النشاط البشري القائمة على الرؤية هي أساس العديد من التطبيقات بما في ذلك المراقبة بالفيديو والرعاية الصحية و المراقبه الامنيه والتفاعل بين الإنسان والحاسوب.

في هذا البحث نقوم بمراجعه تسلط الضوء على التطورات في مناهج التعرف على الأنشطة البشريه باستخدام الحاسوب ودراسه طرق التعرف علي الانشطه البشريه وتقديم رؤى تفصيلية حول الأعمال الحالية والمنهجيات المستخدمة من قبل الباحثين للتعرف على الأنشطة البشرية بالنسبة لتمثيل النشاط وطرق التصنيف. بهدف تقديم نظرة عامة على هذه الأساليب ومقارنتها.

الكلمات المفتاحية: تمييز النشاط البشري, خوارزميات التعلم الالي, مستشعرات الهواتف الذكية, ألاجهز الاستشعار القابلة للارتداء.

\section{1-المقدمه:Introduction-1}

لتمييز النشاط البشري دورًا مهمًا في التفاعل بين الإنسان والعلاقات الانسانيه (human-to-human interaction) ). وحيث ان التعرف علي النشاط البشري يحتاج الي العديد من التطبيقات التي تتطلب التقاعل بين الإنسان والحاسوب والروبوتات لتوصيف السلوك البشري نحتاج الي نظام التعرف على النشاط البشري. من بين الكثير من التطبيقات لتمييز النشاط البشري يبرز السوال المهم وهو "ما هو الإجراء المتخذ لمحاولة التعرف على الأنشطة

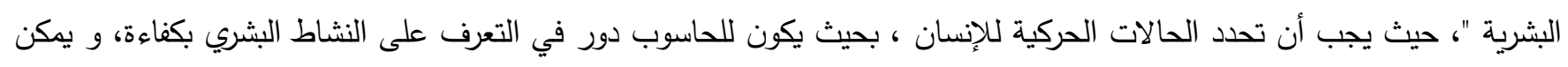
دمج النشاط البشري مع البيانات البيئية المتصورة لتشكيل تمثيل كامل للنشاط البشري. يتم تضمين عدة خطوات في التعرف على النشاط

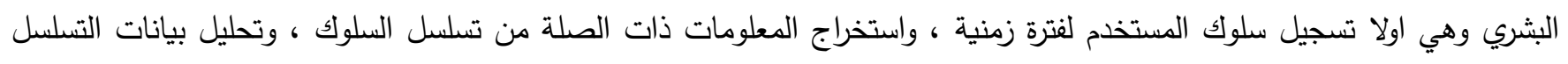
لاشتقاق أنماط محددة. تكثف هذه الأنماط نوعيه النشاط البشري وماذا يجب ان تهئ تقدم له من خدمات كما يمكن استخدام الأنماط لتقديم توصيات أكثر ملاءمة لهم. تلعب الاتصالات والمنتجات الالكترونيه المحموله مثل الهاتف الذكي دورا مهما في التعرف علي النشاط البشري ويوجد حاليًا العديد من التطبيقات للمصلحة العامة حيث هناك حاجة ماسة إلى التعرف علي النشاط البشري على سبيل المثال: في الرعاية الصحية ، لتصميم الأدوية للمرضى ذوي الإعاقة الذين يعانون من مشاكل حركية من خلال المراقبة المستمرة للحركة وأنشطة الحياة اليومية باستخدام أجهزة

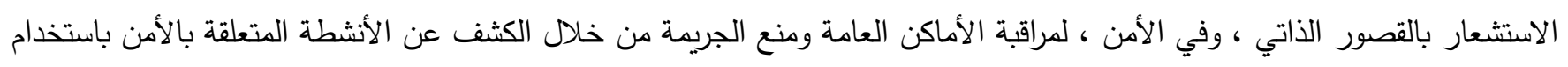

[1]. كاميرات الفيديو

ومع ذلك فان التعرف علي النشاط البشري يعتبر مهمة معقدة بسبب عدة تحديات قد تواجه الباحثين مثل حركة المستشعر وكيفيه تحديده وتحديد الفعاليه بصورة صحيحة بالاضافة الى الخلفية المزدحمة والغير منظمة والتتوع في كيفية تتفيذ الانشطة البشرية المختلفة [3,2]. في هذه الدراسة تم دراسه العديد من البحوث والدراسات حول تقنيات التعرف علي النشاط البشري وتحليل التقنيات الاكثر شيوعا تعات التي تعمل على تمييز والتعرف علي النشاط البشري ودراسة النماذج الحاسوبية والخوارزميات التصنيفية المتعلقة بها. وقد تم تم توضيح ومناقشة ايجابيات وسلبيات كل تقنية منهم. تصني قد تم تتظيم الدراسة عل النحو التالي: القسم الثاني باختصار يقدم كيفية التعرف على النشاط البشري وكيفية تطبيقه في عدة مجالات, اما القسم الثالث فيصف انواع الاستشعار والتقنيات المستخدمة في انظمة تمييز النشاط البشري,القسم الرابع يناقش التحديات التي تواجه 
مصمميي انظمه التعرف علي النشاط البشري والقسم الخامس يوضح فيه الدراسات المتعلقه بطرق تمييز النشاط البشري التي تستخدم خوارزميات التعلم الالي والقسم السادس يوضح فيه التحديات لتصميم نظام التعرف علي النشاط البشري واخيرا يختتم القسم السابع بالاستتتاجات.

\section{2- تمييز النشاط البشري}

تعرف عملية تمييز النشاط البشري بانها القدرة على تفسير حركة جسم الانسان عبر اجهزة الاستشعار وتحديد نوعية هذا النشاط

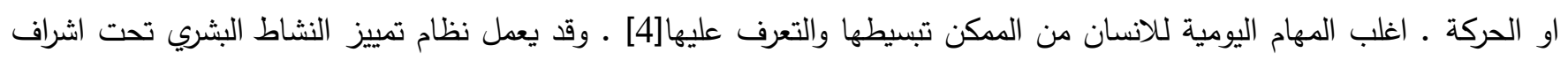
متخصصين او حتى بدون اشراف [5] حيث يتطلب الاشراف على هذا النظام بعض التدريب المسبق باستخدام بيانات معينة, اما عملية استخدام هذا النظام بدون اشراف فيتم ذلك بالاستعانة بمجموعة من القوانين خلال عملية الانشاء والتطوير [6]. لهذا فان دراسه و تصميم انظمه التعرف علي النشاط البشري له اهمية كبيرة في البحوث العلمية الحديثة حيث يدخل في عدة مجالات

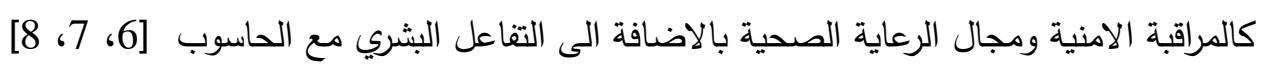
لقد تم اعتماد نظام التمييز البشري في عدة انظمة مثلا للمراقبه الامنيه كمراقبة الاماكن العامة والبنوك والمطارات,حيث قدمت الكثير من لن البحوث بتصميم نماذج تقوم بالتنبؤ بالنشاط البشري للنع حصول الجرائم والانشطة الخطرة في الاماكن العامة [9,5]. اما عند استعراض ولئه المراجع المتعلقة بالرعاية الصحية فان اغلبها قام باستخدام انظمة التعرف علي النشاط البشري المتوفرة في انظمة الرعاية الصحية في

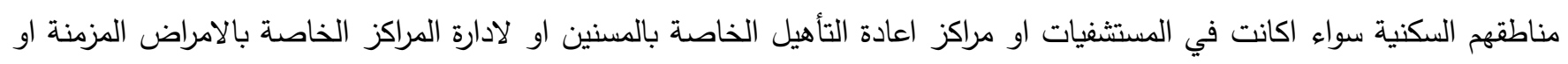
الوقاية منها. وكذلك قد تم دمج هذا النظام مع ما يسمى بالمنازل الذكية المخصصة لكبار السن لتتبعهم ومراقبة تحركتهم وانشطتهم [10],

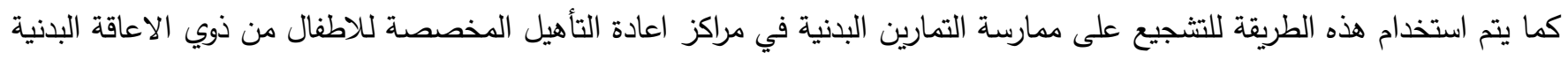
, او لاعادة تأهيل المرضى بعد تعرضهم للسكتة الدماغية , والمرضى الذين يعانون من اي خلل وظيفي او تباطؤ حركي غير ذلك استخدم هذا النظام في عملية مراقبة المرضى في منازلهم, كمراقبة وحساب مقدار الطاقة المبذولة لتقليل السمنة المرضية وكذللك مراقبة صور المرضى وتحركاتهم وتحميلها على الحاسوب من اجل تحليلها . ان اي نظام لتمييز النشاط البشري HAR يتكون من اربع مراحل اساسية، كما موضح في في الشكل (1)[16]: الاستشعار: الاستشعار هو المسؤول عن تجميع بيانات المستشعر من المصادر المتوفرة ومعالجتهم. بشكل عام, يجب اجراء عملية تكييف للاشارة كتقليل الضوضاء او رقمنة الاشارة وتحويلها من تماثلية الى رقمية وتضخيم الاشارة من اجل تكييف الاشارة

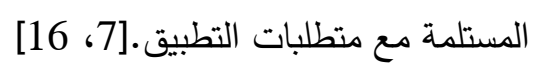

استخلاص الميزات: في عمليه استخلاص الميزات تتم عملية استخلاص البيانات للحصول على مميزات ضرورية تصف البيانات وتسمح بتمثيل وتوضيح الحدث الاساسي بصورة افضل, يتم تحويل هذه البيانات المستخلصة الى مدخلات لخوارزمية

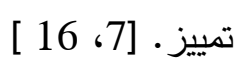

التمييز :في مرحله التمييز تستخدم خوارزميه التعليم , اما لتعلم ودراسة النموذج الحالي او التتبؤ بنشاط العينات الجديدة عند توفر

[7] 


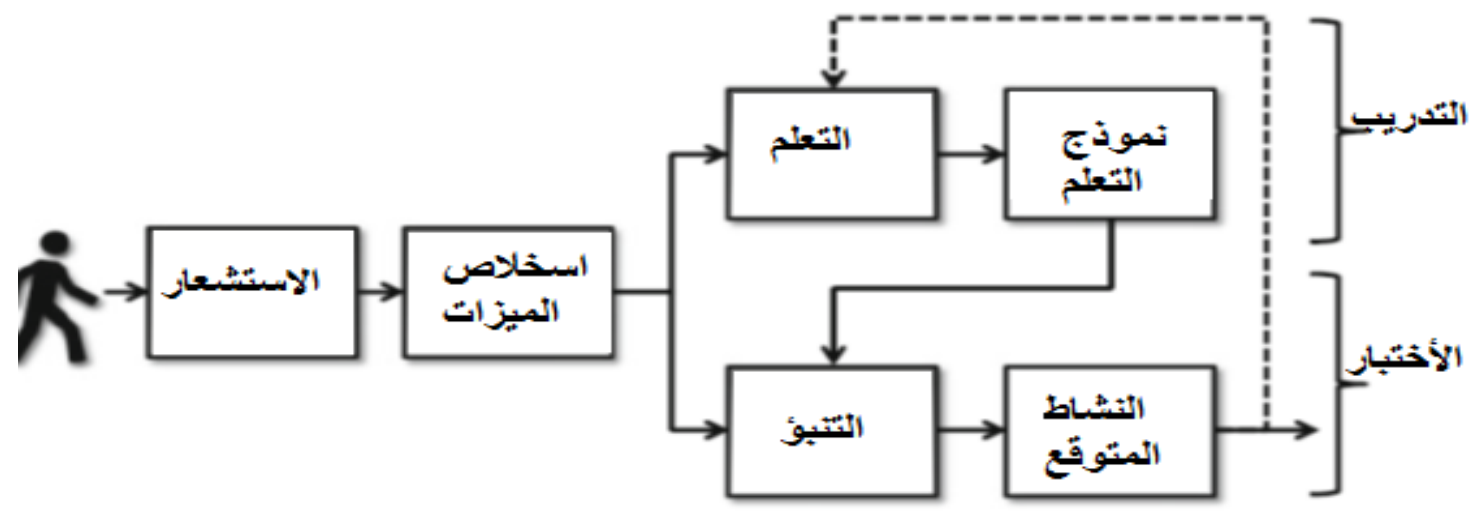

الثكل1.خطوات تمييز النشاط البشري مع مراحله الاربعة الرئيسية [16]

2-2 1الانشطة البثرية

نظام تمييز النشاط البشري يعتمد على مجموعة من الانشطة التي يجب دراستها والتعرف عليها لتاثيرها المباشر على طريقة تصميم وتتفيذ النظام. لهذا السبب, يتم تصنيف هذه الانشطة الى فئات لتسهيل عملية اختيار الالية المناسبة لفهم وتمييز النشاط البشري. يتم تصنيف الانشطة البشرية تبعا لمدتهم ودرجة تعقيدهم ونوع النشاط, الجدول(1) يوضح التصنيف تصنيف الانشطة تبعا لمدتها وتعقيدها

جدول 1 . تصنيف الانثطة البشرية بالاعتماد على مدتها وتعقيدها]

\begin{tabular}{|c|c|c|}
\hline الامثلة & نوع النشاط & المدة/ التعقيد \\
\hline تلويح اليدين/ الايماء بالرأس/ الضحك & 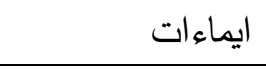 & \multirow[t]{2}{*}{ الاحداث القصيرة } \\
\hline من الوقوف للجلوس/من التمدد للجلوس & 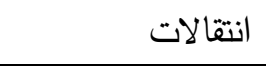 & \\
\hline الوقوف/ الجلوس/ القراءة & ثابتة & \multirow{2}{*}{$\begin{array}{r}\text { الاحداث الاساسية } \\
\text { Basic Activities(BAs) }\end{array}$} \\
\hline المشي/ الركض/ ركوب الدرجات & ديناميكي(متحرك) & \\
\hline الطبخ/ التجميع/ التاثيث/ حمل الاوزان & متعدد الانشطة & \multirow{2}{*}{$\begin{array}{c}\text { الاحداث المعقده } \\
\text { Complex Activities(CAs) }\end{array}$} \\
\hline التكلم/ الرقص/ العناق & متعدد المستخدمين & \\
\hline
\end{tabular}

كذلك من الممكن تصنيف الانشطة بالاعتماد على نوعها, وبشكل عام هذه المجموعة من الانشطة متصلة بالعديد من التطبيقات

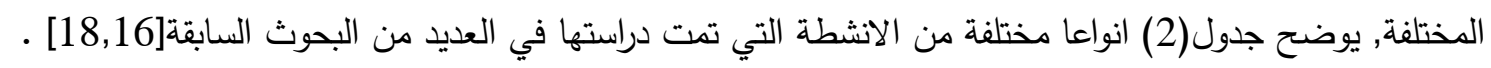

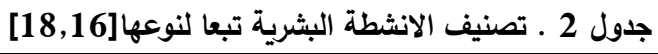

\begin{tabular}{|c|c|}
\hline 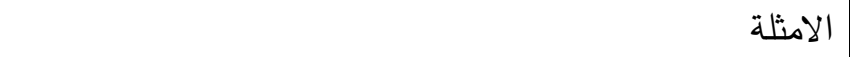 & التطبيق \\
\hline مشاهدة التلفاز ركوي الملابس, تتاول الطعام, الاستحمام, التظظيف & الانشطة اليومية \\
\hline المشي, ركوب الدراجة, الوقوف, الاستلقاء, السقوط & 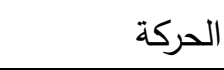 \\
\hline القغز , رفع الاثقال, التسلق, السباحة & الرياضة/ اللياقة \\
\hline الاتصال الهاتفي,المراسلة, التكلم, الغناء & 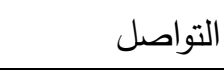 \\
\hline التسكع, المطاردة, الإثراف, المطاردة & الامان/ المراقبة \\
\hline
\end{tabular}




\section{3-تقنيات الاستشعار}

بشكل عام, تقنيات الاستشعار تلعب دورا مهما في التعرف وتمييز النشاط البشري, الشكل(2) يوضح كيفية التعرف على النشاط البشري, حيث عندما يقوم الانسان بتحريك اي جزء من جسمه سواء يد او قدم او رأس ستقوم اجزة الاستشعار بالتقاط هذه الحركة ونقلها الى محرك بحث لتمييز وتحليل المعلومات وتحديد نوع الحركة التي قام بها الشخص. وليعد تحديد واختيار نوع جهاز الاستشعار الصحيح احد اهم العناصر التي يجب مراعتها عند تصميم انظمة تمييز الانشطة البشرية, حيث هناك عدة اجهزة استشعار قد تم اتكثافها لاستخلاص المعلومات المتعلقة بالتمييز • ويتم باستخدامها قياس وتحديد عدة سمات بما في ذلك العلامات الحيوية (مثل درجة حرارة الجسم ومعدل ضربات القلب وضغط الدم) والاشارات البيئية (مثل شدة الضوء ومستويات الصوت) والحركة (مثل التسارع والسرعة) وتحديد الموقع(مثل باتل

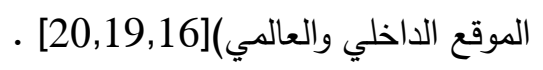

المستشعر بالنسبة للمستخدم, يتم تصنيف اليات الاستشعار بالاعتماد عندما تكون المستشعرات قابلة للارتداء او متصلة بجسم معين.

يتم توضيح هذين التصنيفين في الشكل 2 مع ذكر امثلة عن كلا النوعين. [16، 19

(Ambient Sensors) -

أجهزة الاستشعار المحيطة ، والمعروفة أيضًا باسم المستشعرات الخارجية أو البيئية ، هي مجموعة من الأجهزة الموجودة في

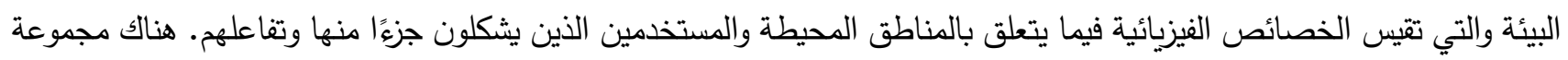
واسعة من أجهزة الاستشعار المحيطة مثل الميكروفونات وكاميرات الفيديو ومستشعرات الوجود ومقاييس الحرارة وأجهزة استشعار العمق. تم بالفعل استخدام العديد من أجهزة الاستشعار هذه في HAR. على الرغم من اجهزه الاستشعار المحيطه تقدم معلومات

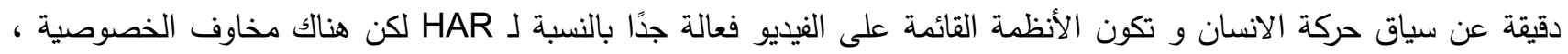
على سبيل المثال ، عند استخدامها في بيئات المنزل لأن الناس بشكل عام غير مرتاحين بشأن استمرار مراقبتهم. ومن انواع ي اجهزة

$$
\text { الاستشعار المحيطة }
$$

> مستشعر الوجود (Presence sensors) :تكتشف أجزة استشعار الوجود مسافة الجسم أو غيابه أو وجوده. / على سبيل المثال ، شخص ما في المطبخ أثناء الصباح أثناء تشغيل آلة القهوة أن الشخص يقوم بإعداد وجبة الإفطار .

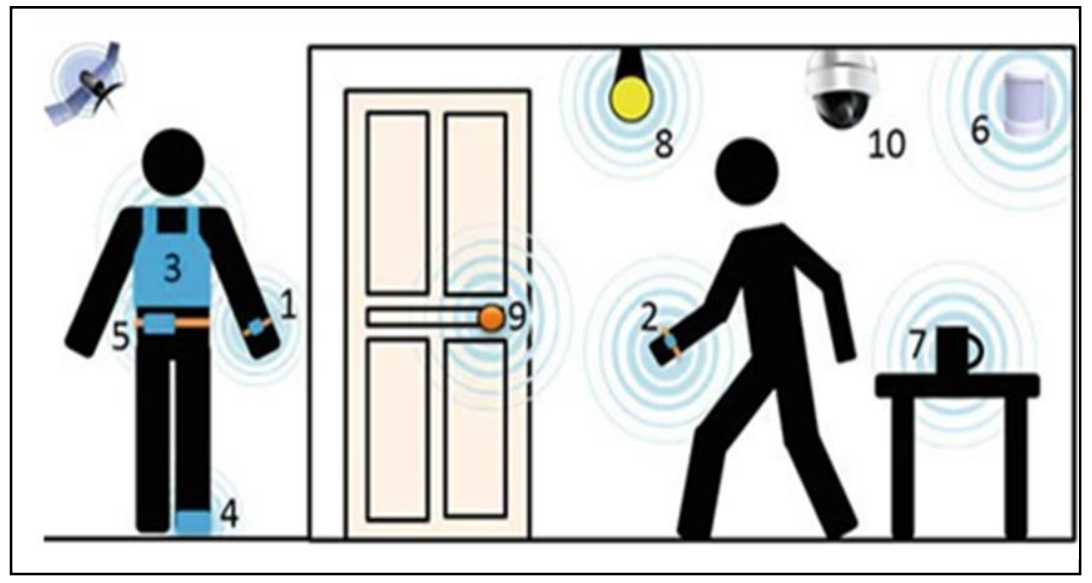

مستشعرات قابلة للارتذاء 1. 1 ساعة استشعار الموقع

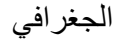

2. 2 المستشعر الداخلي

3. سترة العلامات الحيوية 4. عداد الخطى ستى العلات 5. هاتف الذكي

6هزة الاستشعار المحيطة

6.مستشعر الوجود

7. مستشعر القرب منشع الوحود

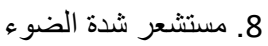

9. مستشعر فتح الباب

10. الكامير ا الفديوية

الثكل 2 ـ مثال على اجزة الاستثعار للمجال المحيطو القابلة للاتداء [16] 
مستشعر القرب (proximity sensors ) : مستشعر القرب جهاز إستشعار قادر على اكتثاف وجود الأجسام القرببة منه بدون حدوث أي اتصال جسدي./ علي سبيل المثال أجهزة المحمول والشاشات التي تعمل باللمس وتقترب من الوجه

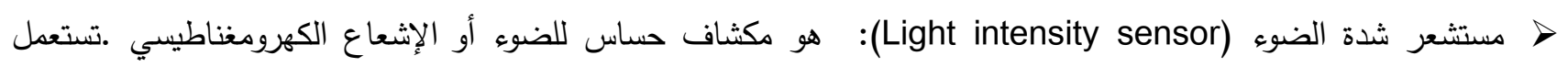
المجسات الضوئية في القياسات الضوئية المختلفة أو لتشغيل أجهزة أو أدوات معينة، او لتحسس حركه شخص مثل مثل قيامه بفتح الباب وقفله > مستشعر فتح الباب (Open door sensor): وهو مستشعر يعرف ايضا باسم الباب التلقائي ، هو باب يُفتح تلقائيًا ، عادةً عند استشعار اقتراب شخص ما. الكاميرا الفديوية (d) d(Video camera sensor : يتم استخدام الكاميرات لتحليل الحركة البشرية القائمة على الرؤية بدون علامات.

(Wearable Sensors) - مستشعرات قابله للارتداء تستخدم أجهزة الاستشعار القابلة للارتداء لجمع الإشارات مباشرة من المستخدمين. يتم ربطها عادةً بأجزاء مختلفة من الجسم مثل الخصر والمعصم والصدر والساقين والرأس ولكنها أيضًا متصلة بالملابس ويتم تضمينها في ملحقات أخرى للاستخدام المنتظم مثل الساعات والنظارات أو الهواتف المحمولة. ومن انواع مستشعرات قابله للارتداء: ساعة استشعار الموقع الجغرافي ( GPS sensor watch ) : ساعة GPS عبارة عن جهاز به مستقبل GPS مدمج يتم ارتداؤه كوحدة واحدة مربوطة بمعصم بطريقة السوار • يمكن أن تحتوي الساعة على ميزات وإمكانيات أخرى حسب الغرض

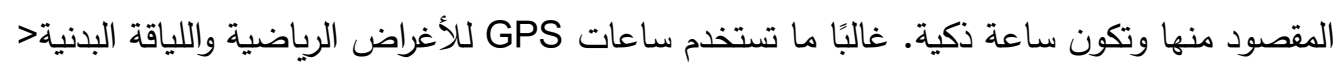
> المستشعر الداخلي (inertial sensor) :ي جهاز إلكتروني يقيس ويبلغ القوة المحددة للجسم ، ومعدل الزاوية ، وأحيانًا اتجاه الجسم

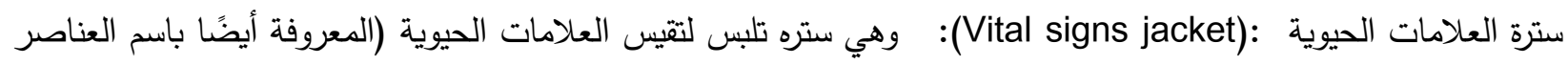
الحيوية) هي مجموعة من أربع إلى ست علامات طبية مهمة تشير إلى حالة وظائف الجسم الحيوية (الحفاظ على الحياة). يتم أخذ هذه القياسات للمساعدة في تقييم الصحة البدنية العامة للشخص ، وإعطاء أدلة على الأمراض المحتملة ، وإظهار التقدم نحو الشفاء

عداد الخطى (pedometer) : عداد الخطى هو جهاز يحسب عدد الخطوات أو المسافة التي يقطعها أحد مشي : هاء هاتف الذكي (Smart phone) : هو مصطلح يطلق على فئة من الهواتف المحمولة الحديثة التي تستخدم نظام تشغيل منطور،

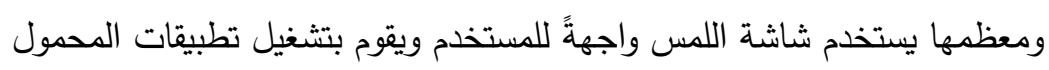

تستخدم اجهزه الاستشعار في انظمه التعرف علي النشاط البشري حيث تاخذ منها المعلومات وتحول من قبل مصمم نظام التعرف علي النظام البشري الي قيم رقميه لكل انظمه الاستشعار وتكون منها قاعده بيانات، وتعتبر قاعده البيانات ادخال الي خوارزميه التمييز والتعرف علي النشاط البشري، كمثال علي ذلك، الثكل (3)، حيث يستخدم الهاتف الذكي المحمول بواسطه الانسان كجهاز استشعار، و و عن طريق المستشعرات الموجوده بالهاتف الذكي والبرنامج المصدم من قبل مصدم نظام التعرف علي النشاط البشري، يتم تحول اي 
نشاط بشري الي قيم رقميه تخزن في قاعده البيانات، وتقسم هذه البيانات الي بيانات تدريب وبيانات اختبار . وهذا العمليه تتطبق علي

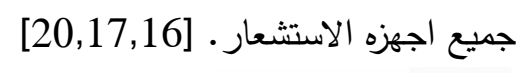

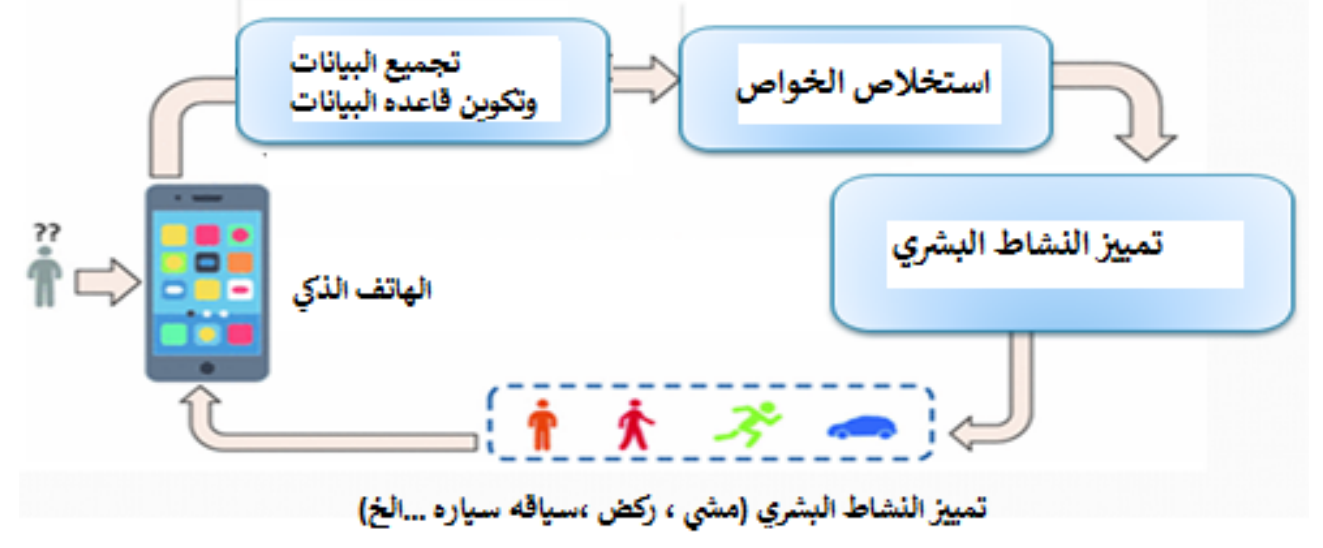

الثكل (3) : تمييز النشاط البشري باستخدام المستثعر الهاتف الذكي[16]

4-التحديات التي تواجه مصمي نظام تمييز النشاط البثري تصميم نظام تمييز النشاط البشري يعتمد على الانشطة التي يجب ان يقوم بتمييزها والتعرف عليها, حيث انه من المكن ان تؤثر

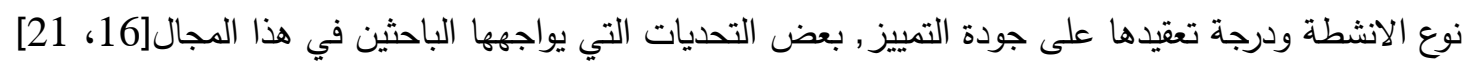
• • • ب كيفية اختيار السمات المراد قياسها. • كيفية بناء نظام التمييز ببيانات قابلة للنقل بين اكثر من جهاز الكتروني. • كيفية استخراج الميزات وتصميم طرق الاستدلال.

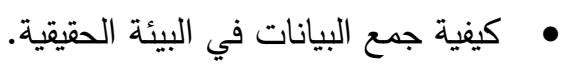
• كيفية التعرف على المستخدمين وعلى انشطة المستخدمين الجدد دون الحاجة الى اعادة تدريب النظام.

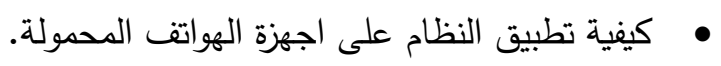

5- الخوارزميات المستخدمه لتصميم نظام تمييز النشاط البشري

استخدمت الخوارزميات التقليديه وخوارزميات الذكاء الاصطناعي والتعلم الالي (Machine learning) في تصميم انظمه تمييز النظام البشري ومع التطور التقني السريع مثل الهواتف الذكية والساعات الذكية واجهزة الاستشعار القابلة للارتداء, ومع امكانيه تزويد هذه الاجزة بتقنيات وتطبيقات الذكاء الاصطناعي للتتبؤ بالنشاط البشري, بالاعتماد على التسارع الحاصل لاشارة المستشعر اصبحت امكانيه تصميم انظمه تمييز النشاط البشري ممكناـ[20] الهدف الاساسي من انظمة تمييز النشاط البشري هو استخدام نموذج التعلم الالي الذي يمتاز بالدقة العالية لتنبأ النشاط البشري, وهناك

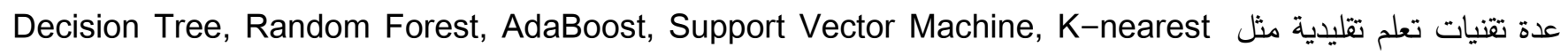


neighbor, Naïve Bayes, الالي لتمييز النشاط البشري كان كفوءا جدا , عند دراسة الكثير من الابحاث وجد ان هناك استراتجيتين مختلفتين لبناء نموذج التعلم الالي

• النموذج التقليدي(Traditional model ) : في هذا النموذج يتم جمع البيانات من المستشعرات, ثم استخلاص الميزات, بعدها يتم استخدام احدى التقنيات الذكائية واخيرا تستخرج قائمة بالنشاطات البشرية. نموذج التعلم الالي ( Machine learning ): في هذا النموذج يتم جمع البيانات, واستخراج الميزات, ثم استخدام احدى تقنيات التعلم الالي, واخيرا استخراج قائمة بالنشاطات البشرية. [22,17].

ونستعرض هنا بعض الدراسات والبحوث في مجال تمييز النشاط البشري، حيث ذكر الباحث Kantoch في سنه 2012 انه من خلال قراءة وتحليل عدد كبير من البحوث, وجد ان 56.25 \% من الابحاث تركز على استخدام الخوارزميات التقليدية في التصنيف,

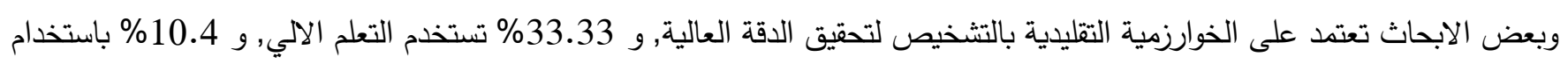
الخوارزميات (التعلم الالي والخوارزميات التقليدية) لفحص واختبار النظام المقترح [24,23] بعتية. حيث قام [Arifoglu et al. [25 بعمل مقارنة بين ثلاث انواع من الثبكات العصبية الحديثة, والتي تعد من خوارزميات التعلم الالي,

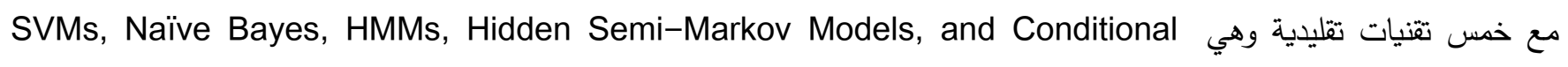
Random Fields (CRFs) انشطة الحياة اليومية مثل النوم والطهي ومغادرة المنزل وما الى ذلك باستخدام اجهزة استشعار موضوعة في المنزل لمدة تقارب الشهر , النتائج اظهرت بان التعلم الالي منافس متميز لانواع التعلم التقليدية.

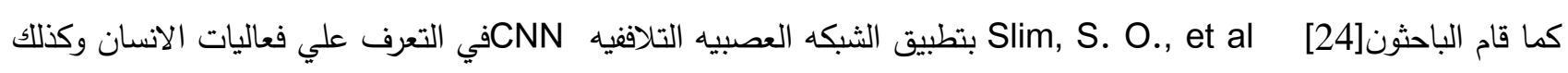

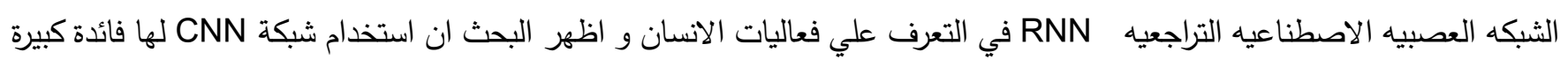
للتعرف علي فعاليات الانسان افضل من شبكة RNN قام الباحثون [Hayashi et al. [27 باستخدام الثبكه العصبيه العميقه Deep Neural Networks (DNN) واقتراح طريقة جديدة تسمى دالة التوزيع التراكمي التجردية (ECDF) لتمثيل البيانات الناتجة من اجهزة الاستشعار لتحسين كفاءة استخراج الميزات[27,24].

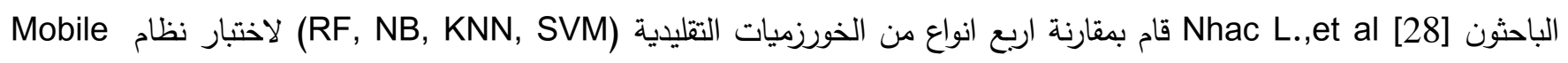

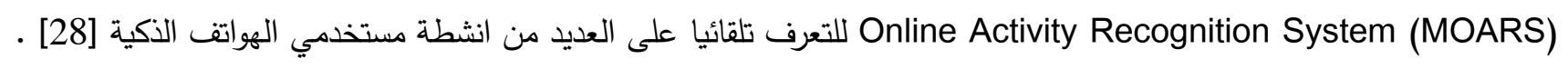
الباحثون[Kwapisz,J.R. et al [29 ركزو على تطوير مجموعة من قواعد البيانات الخاصة التي جمعت باستخدام اجزة الهواتف

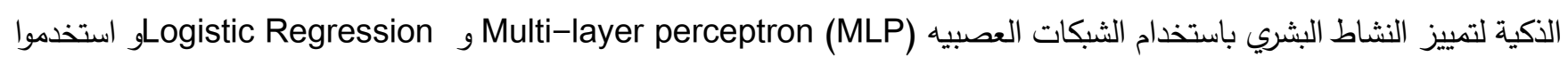

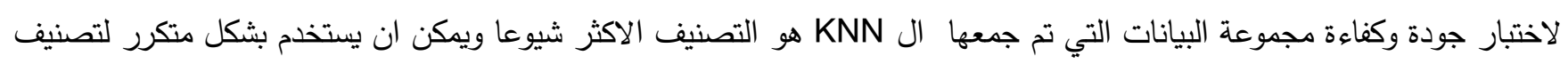

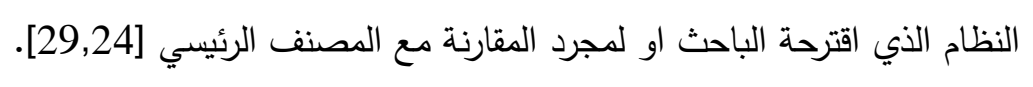
طور باحثون Bayat et al تصنيف جديد يعتمد على الدمج بين خوارزميات التصنيف التقليدية كما في [30] وهو مايسمى بالخوارزميات الهجينه . 
Brezmes,T. et al, [41] الحركات البشرية. لكنه لم يوفر معالجة مركزية للبيانات ولهذا فان مراقبة التحركات البشرية تتم بصورة لامركزية ولذلك يتطلب برنامج

$$
\text { اضافي لكي يسجل ويبلغ عن التغييرات والتحركات البشرية. }
$$

Sun, L.,et al, [42]

Decision Tree, Naïve Bayes, Random Forest, Logistics Regression, وتمييز النشاط البشري باستخدام خوارزميات RBF Network, Support Vector Machine ان الشبكات العصبية هي نموذج برمجي مستوحى من الناحية البايولوجية للانسان ويمكن للحاسوب من التعلم من بيانات المستشعر والتعلم الالي, وهو مجموعة قوية من التقنيات للتعلم في الثبكات العصبية. الشبكات العصبية NN حققت متوسط دقة مقبول. التعلم الالي له بنى مختلفة مثل الثبكه العصبيه التلاففيه(Convolution Neural Network CNN ) والشبكه العصبيه التراجعيه ولقد وجد الباحثون ان متوسط الدقة لجميع بيانات التعلم الالي متقاربة بالغالب ولكن الاكثر (Recurrent Neural Network RNN) استخداما هي CNN, جدول (3) يوضح اهم الطرق الذكائية المستدمة في تمميز النشاط البشري وايجابياتها وسلبياتها وذقة كل منها.

\begin{tabular}{|c|c|c|c|c|c|}
\hline الدقة & السلبيات & الايجابيات & البحث منهاج & الخوارزميات & الباحثون \\
\hline $98 \%$ & 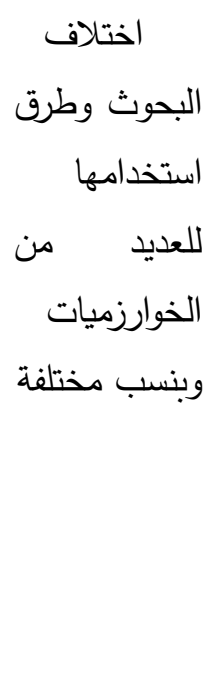 & 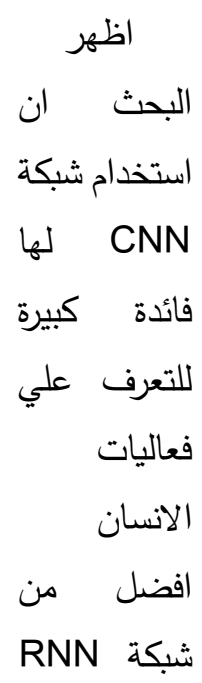 & 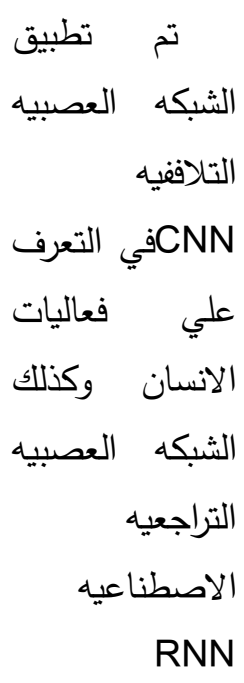 & 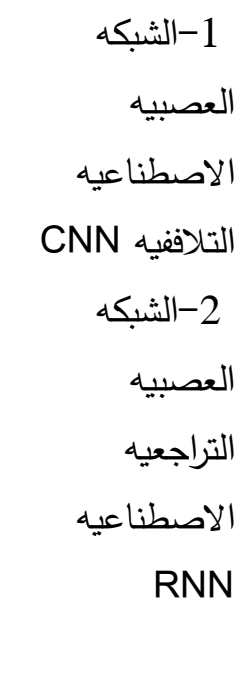 & $\begin{array}{l}\text { Slim, S. O., } \\
\text { [24],2019et al, }\end{array}$ \\
\hline$\% 95$ & ليت اليتم & 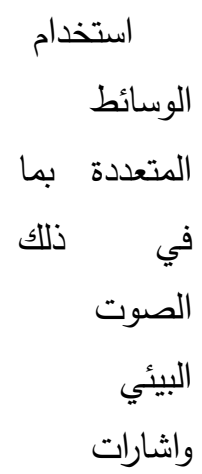 & 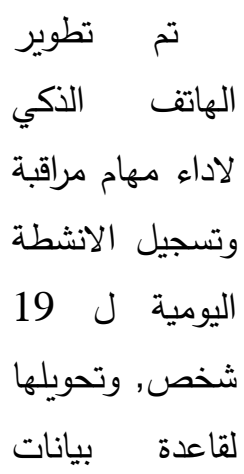 & $\begin{array}{r}\text { الشبكه العصبيه } \\
\text { deep neural } \\
\text { networks } \\
\text { (DNNs) }\end{array}$ & $\begin{array}{l}\text { Hayashi, T, } \\
\text { et al, } \\
2008 \\
\text { [27] }\end{array}$ \\
\hline
\end{tabular}




\begin{tabular}{|c|c|c|c|c|c|}
\hline & الانشطة في الطلق. & التسريع ثلاثي & تتكون من اشارات & & \\
\hline$\% 95.5$ & 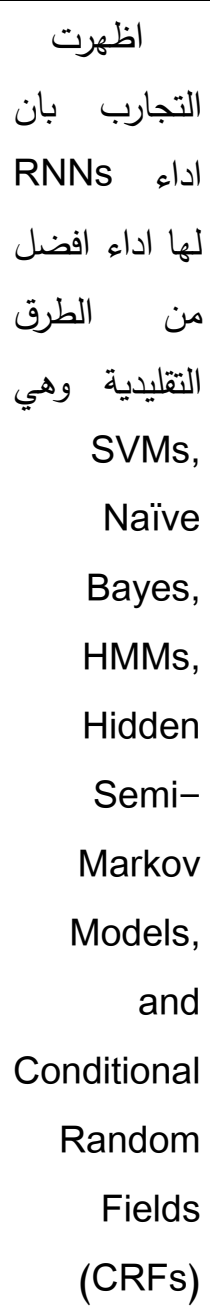 & 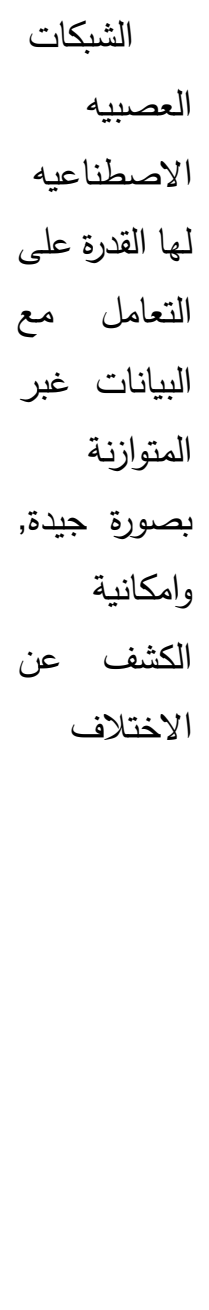 & فيلاث & 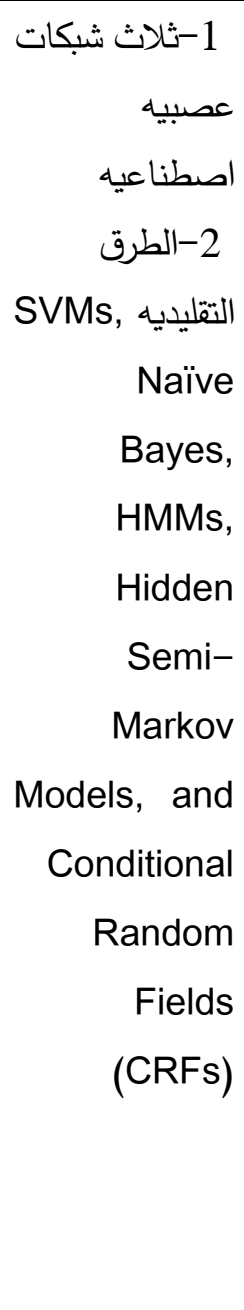 & $\begin{array}{l}\text { Arifoglu, D., } \\
\text { et al, } \\
{[25], 2017}\end{array}$ \\
\hline$\% 91.15$ & 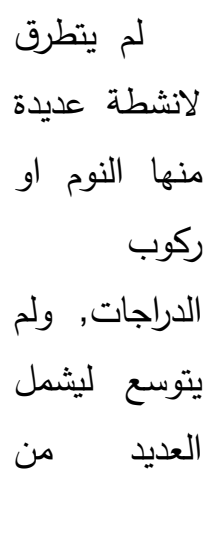 & ثبن بلات & 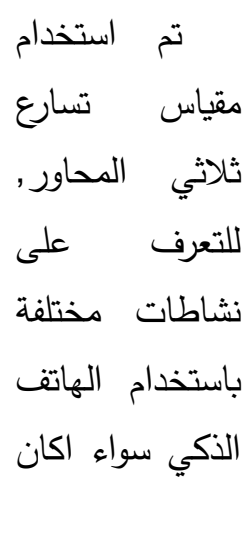 & $\begin{array}{c}\text { Digital low- } \\
\text { pass filter. }\end{array}$ & $\begin{array}{l}\text { Bayat, A., et } \\
\text { al } \\
{[30], 2014}\end{array}$ \\
\hline
\end{tabular}




\begin{tabular}{|c|c|c|c|c|c|}
\hline & العمرية. & 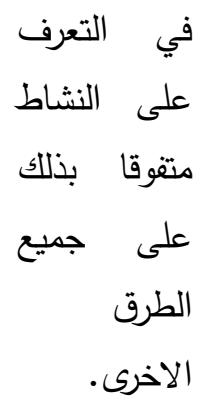 & فيب اليد او في & & \\
\hline$\% 89$ & 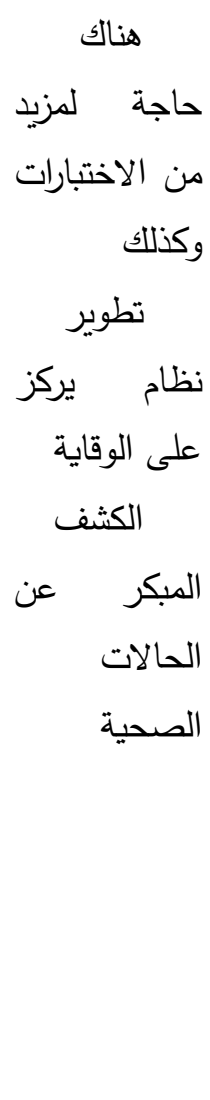 & تالتي & 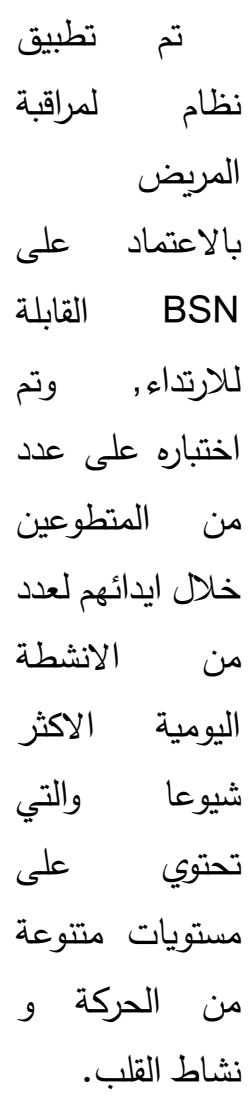 & BSN & $\begin{array}{l}\text { Kantoch, E., } \\
\text { et,al, } \\
\text { [23] } 2012\end{array}$ \\
\hline$\% 91.1$ & 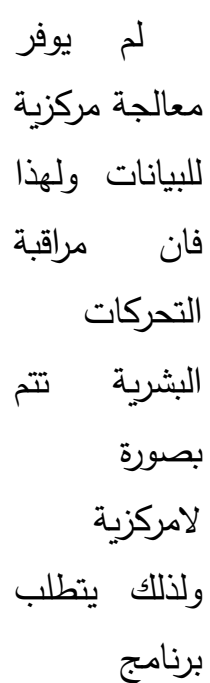 & 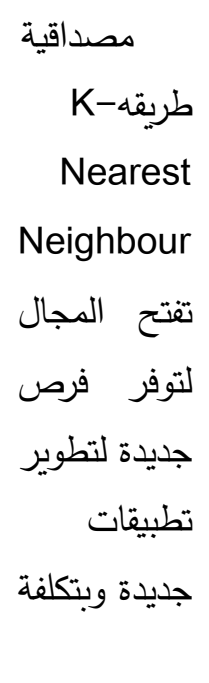 & 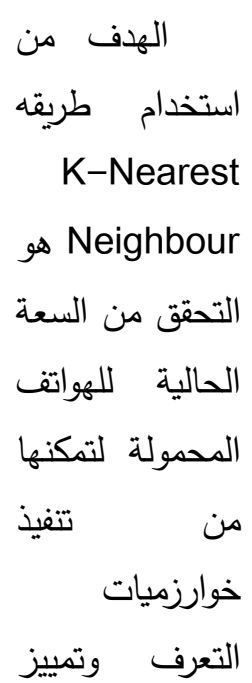 & $\begin{array}{l}\text { K-Nearest } \\
\text { Neighbour }\end{array}$ & $\begin{array}{l}\text { Brezmes, T. } \\
\text { et al, } 2009 \\
{[41],}\end{array}$ \\
\hline
\end{tabular}




\begin{tabular}{|c|c|c|c|c|c|}
\hline & 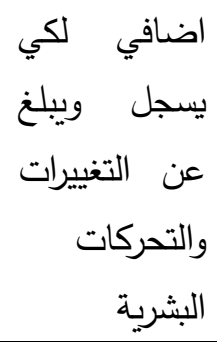 & متخفة نوعا & التصنيف الانماط اللازمة & & \\
\hline $97.7 \%$ & 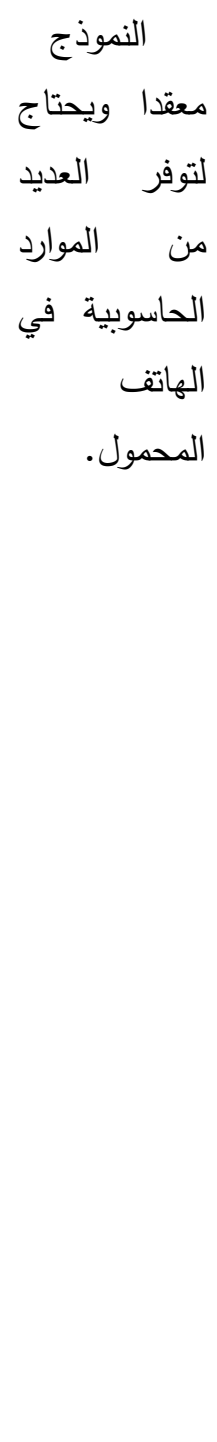 & 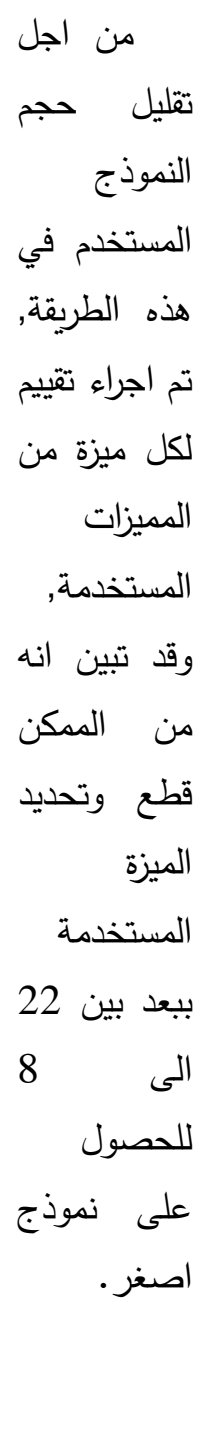 & 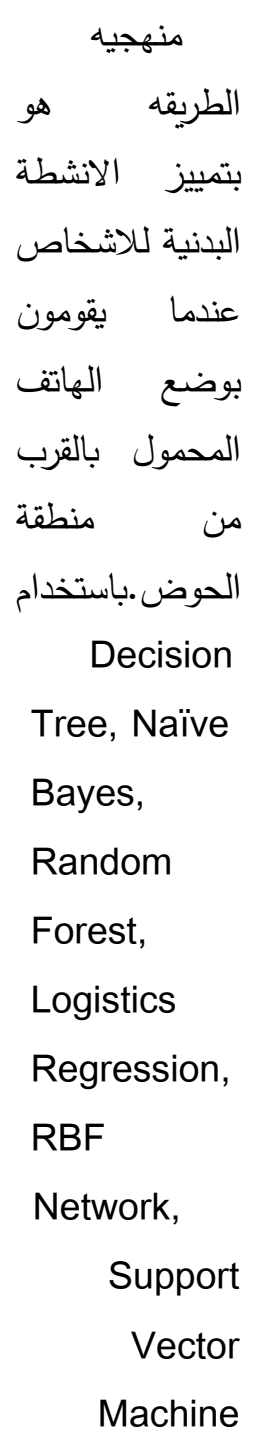 & $\begin{array}{r}\text { Decision } \\
\text { Tree, Naïve } \\
\text { Bayes, } \\
\text { Random } \\
\text { Forest, } \\
\text { Logistics } \\
\text { Regression, } \\
\text { RBF } \\
\text { Network, } \\
\text { Support } \\
\text { Vector } \\
\text { Machine }\end{array}$ & $\begin{array}{l}\text { Sun, L.,et } \\
\text { al., } \\
\text { [42], } 2011\end{array}$ \\
\hline$\% 90$ & فل الطرق التقليديه & التشرف النيده في & النتخدام & 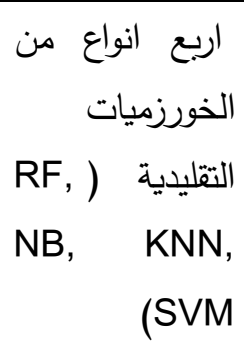 & $\begin{array}{l}\text { Nhac,L., at } \\
\text { el, } 2018 \\
\text { [28] }\end{array}$ \\
\hline
\end{tabular}




\begin{tabular}{|c|c|c|c|c|c|}
\hline & تكون البيانات & & & & \\
\hline$\% 94.6$ & 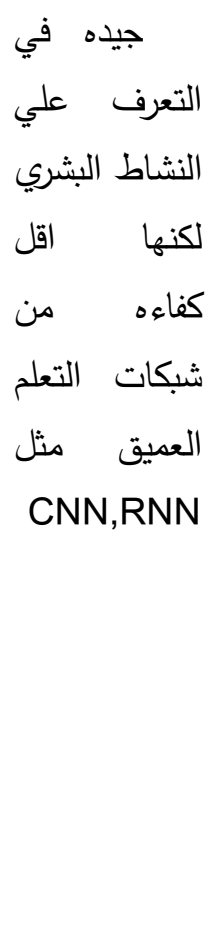 & 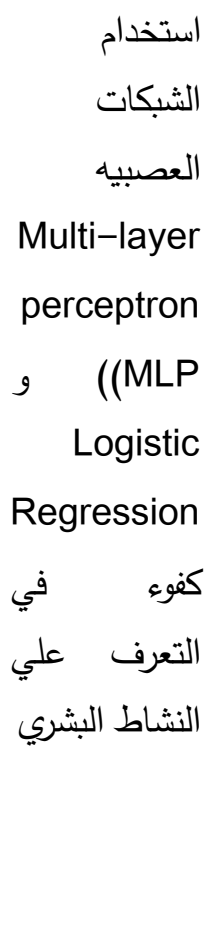 & 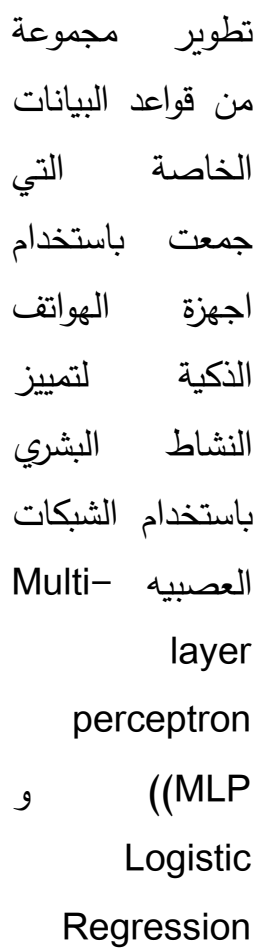 & $\begin{array}{r}\text { الثبكات } \\
\text { Multi- العبيكات } \\
\text { layer } \\
\text { perceptron } \\
\text { ((MLP } \\
\text { Logistic } \\
\text { Regression }\end{array}$ & $\begin{array}{l}\text { Kwapisz,J.R. } \\
\text { et al } \\
2011 \\
\text { [29] }\end{array}$ \\
\hline
\end{tabular}

وبهذا نستخلص بان خوارزميات التعلم الالي تمتاز بانها خوارزميات خطية, حيث انه بالاككان تمثيلها بواسطة عقدة واحدة فقط تقوم بتحويل المدخلات خطيا الى مخرجات. سابقا أطلق على التعلم الالي بالثبكة العصبية الاصطناعية, والتي تمتاز باستخدامها عدة عقد بأه تتظم على شكل الشبكات العصبية لتمثيل كيفية عمل العقل البشري. وكلما زادت عدد العقد والطبقات بالشبكات العصبية, زادت قدرات التعلم الخاصة بها[31].

على الرغم من استمرارية استخدام مصطلح الشبكات العصبية, فان شبكات التعلم الالي اليوم تمثل تدفق المعلومات عبر العقد والتي تثبه كيفية تدفق المعلومات الى الدماغ البشري عبر الخلايا العصبية, وتميل الابحاث الى استخدام التعلم الالي بديلا عن التعلم التقليدي. ووفقا للعديد من البحوث التي تمت دراستها, فان خوارزميات التعلم الالي ظهر بين عام 2014 الى عام 2018, ولكنه بعد عام 2014 اصبح رائجا استخدام خوارزميات التعلم الالي اكثر من استخدام الخوارزميات التقليدية في تمييز النشاط البشري[24]. مراقبة التحركات البشرية يمكن بسهولة تصويرها واستخدامها للعديد من الاغراض والتطبيقات المستقبلية. حيث قدم هذا البحث نظام تصنيف لبعض الحركات البشرية الاساسية في الوقت الحقيقي باستخدام الهاتف المحمول مزود بقياس متسارع. وقد حقتت هذه الدراسة الهذف المطلوب منها وهو قياس السعة الحالية للهواتف المحمولة لتنفيذ خوارزميات تمييز الانماط في الوقت الحقيقي لتصيف وتمييز التحركات البشرية. لم يتم توفير معالجة مركزية للبيانات ولهذا فان مراقبة التحركات البشرية تتم بصورة لامركزية ولذلك يتطلب برنامج اضافي لكي يسجل ويبلغ عن التغييرات والتحركات البشرية, مصادقية هذا النهج تفتح مجال جديدا لتطوير تطبيقات جدية وبتكلفة 


\section{Journal of Education and Science (ISSN 1812-125X), Vol: 30, No: 5, $2021(12-29)$}

القدرة المميزة للاستشعار التي تمتاز بها الهواتف الذكية توفر فرصة فريدة لكشف والمراقبة المستمرة للتحركات البشرية, ومع ذلك, تطبيقات الهواتف الذكية يجب ان تمتثل لاستخدام وعادات الانسان بالاضافة الى التقاط اللحظة المناسبة لتساعد على تمييز النشاط البشري, والتي من المحتمل ان تسبب تباينات واختلافات كبيرة. نتيجة لذلك, قد يكون النموذج معقدا ويحتاج لتوفر العديد من الموارد الحاسوبية في الهاتف المحمول. يتم تمييز النشاط البشري عند وضع الهتف المحمول في الجيب بالقرب من منطقة الحوض. وقد اظهرت النتائج ان دقة التمييز قد تصل ال 97.7\%, من اجل تقليل حجم النموذج المستخدم في هذه الطريقة, تم اجراء تقييم لكل ميزة من المميزات المستخدمة, وقد تبين انه من الممكن قطع وتحديد الميزة المستخدمة ببعد بين 22 الى 8 للحصول على نموذج اصغر [42].

6 - القضايا والتحديات

هناك الكثير من التحديات والمعوقات التي تواجه مصممي انظمه التعرف على النشاط البشري منها استخدام اجزة الاستشعار المختلفة لجمع البيانات الاولية للتعرف على الانشطة, و هناك ثلاث انواع من اجهزة الاستشعار : مستشعرات الفذيو , اجهزة الاستشعار البيئية, واجهزة الاستشعار القابلة للارتداء[32]. وتعتبر الكاميرا اجزة استشعار فديوية تحدد وتثبيت باماكن خاصة. ولكاميرات RGB تركيزا اقل بالنسبة لابحاث ال HAR, ربما بسبب تقييدها بالتقاط الصور وحركة الانسان في البية ثلاثية الابعاد. وكذللك التعرف على بلى الثخص وتمييزه عن بيئته يتطلب محددات اكثر بسبب ان العملية تحتاج لمعالجة الية عالية. لهذا تتاثر جودة نظام تمييز النشاط البشري بالوقت الحقيقي. [33] كما ان من المعوقات التي تواجهها المستشعرات القابلة للارتداء هي التقييد والانسداد عند استخدام كاميرا ال RGB. وان العيب الاساسي للمسشعرات القابلة للارتداء هو دقة التعرف, وذلك بسبب ان نظام تمييز النشاط البشري الذي يعتمد على المستشعرات القابلة للارتداء يحتاج لعدة مميزات منها امكانية وضع المستشعرات بالموقع المطلوب بالاضافة الى توزيع هذه المستشعرات على اجزاء الجسم

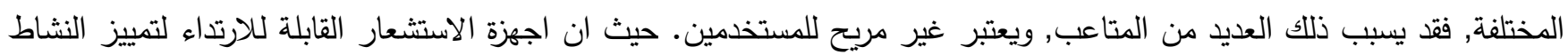
البشري لايمكن ان تكون فعالة فقد ينسى الشخص استخدام المستشعر الخاص به. تمتاز تحديد موقع المستشعر بالنسبة للمستشعرات القابلة للارتداء او مستشعرات الهواتف الذكية بالحساسية الثديدة لتاثيرها الكبير على دقة المعلومات. تعتمد قراءة البيانات الاولية لمقاييس التسارع الموجودة في الهواتف الذكية او المستشعرات القابلة للارتداء على موضع المستشعر او اتجاهه على جسم الانسان. على سبيل المثال تختلف قراءة البيانات المتحركة تماما عندما يمشي الانسان والهاتف لده الذكي في يده او في جيبه. لذلك واجه العديد من الباحثين هذه المشكلة في محاولاتهم لايجاد الحل الامثل [39,35]. في حالة التعلم التقليدي, يجب استخلاص المميزات من بيانات المستشعر الاولية من قبل اي خبير بهذا المجال من اجل تقليل تعقيد البيانات وكذللك جعل الانماط اكثر وضوحا لخوارزمية التعلم. اما في التعلم الالي فله ميزة اساسية وهي امكانيته في استخراج الميزات عالية المستوى من البيانات بطريقة تدريجية ولهذا ليس هناك حاجة للخبرة الميدانية عند استخلاص الميزات. فيما يتعلق بنهج حل المشكلات, تقسم تقنيات التعلم الالي المشكلة الى اجزاء مختلفة ليتم حلها اولا ثم يتم جمع النتائج في المرحلة النهائية بينما يهدف التعلم الالي لحل المشكلة من البداية الى النهاية. كمثال الى ذلك , بالنسبة لمشكلة الكثف عن كائنات متعددة فان تقنيات التعلم الالي مثل نظام Yolov2 تاخذ الصورة كمدخل وتوفر الموقع واسم الكائنات عند الاخراج. .40] ـ من ناحية اخرى فبالنسبة لخوارزميات التعلم الالي الجانبية الاخرى مثل SVM, يلزم وجود خوارزمية للكشف عن الصندوق المحيط اولا لتحديد جميع الكائنات الممكنة للحصول على كائن معين كمدخل لخوارزمية التعلم من اجل تمييز الكائنات المتعلقة به. 
لتقنيات التعلم الالي متطلبات محددة ليتم تطبيقها وهي توفر الالات المتطورة وهو عكس ما موجود بخوارزميات التعلم التقليدي

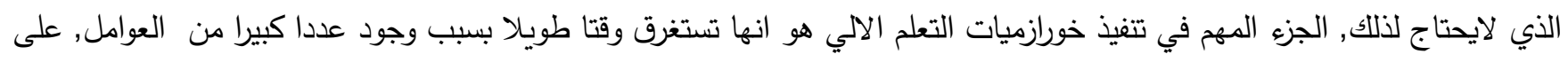
سبيل المثال, تستغرق خوارزمية Popular Deep Residual Networks حوالي اسبوعين للتدريب تماما من الصفر , بينما يستغرق تدريب خوارزمية التعلم الالي التقليدية مثل KNN بضع ثوان الى بضع ساعات. في مرحلة الاختبار يكون الامر معاكسا تماما. تستغرق

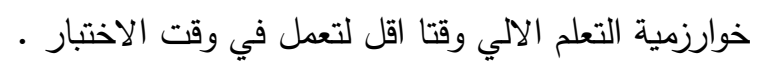

7- تقييم اداء نظام التعرف علي النشاط البشري

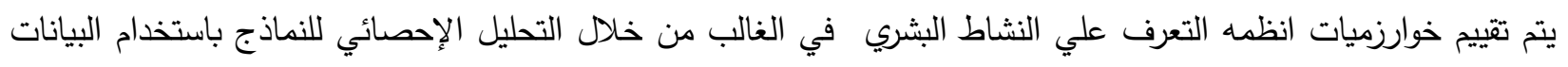
التجريبية المتاحة. الطريقة الأكثر شيوعًا هي مصفوفة الارتباك (confusion matrix) التي تسمح بتمثيل أداء الخوارزمية من خلال تحديد أنواع الأخطاء بوضوح (الإيجابيات والسلبيات الخاطئة) والعينات المتوقعة بشكل صحيح على بيانات الاختبار ـ منه ، يمكن أيضًا استخراج مقاييس مختلفة مثل دقة النموذج والحساسية والدقة بالإضافة إلى ذلك ، يمكن أن تدعم المؤشرات النوعية المقارنة الأخرى ، مثل الإيل عدد الأنشطة المتاحة وسرعة التتبؤ واستهلاك الذاكرة ، ولتقييم الاداء يتم أخذ هذه القيم بافتراض أن الفئة أ هي فئة الاهتمام أو الحالة

الإيجابية. [17,16]

• الإيجابيات الحقيقية (TP): عينات فعلية للفئة A تم توقعها بشكل صحيح كفئة أ

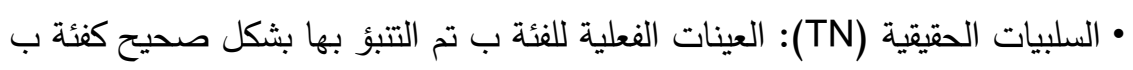
• الإيجابيات الكاذبة (FP): عينات فعلية من الفئة ب تم توقعها بشكل غير صحيح كفئة أ

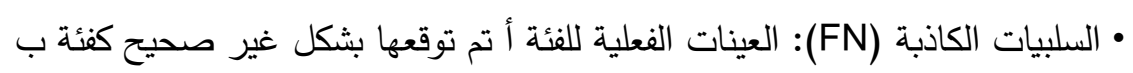

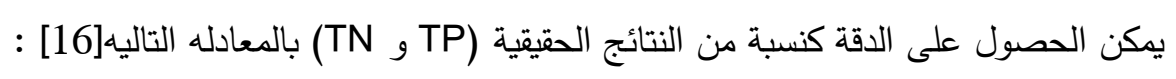

الدقه =

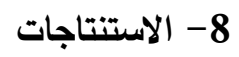

يستعرض هذا البحث احدث التقنيات في التعرف وتمييز النشاط البشري, والتي لها دور كبير في تمييز والتقاط حركة كل جزء من جسم الانسان ثم يتم نقلها لاحدى محركات البحث لتمييزها وتحليلها وتحديد نوعها, تم ذكر وتصنيف العديد من اجهزة الاستثعار

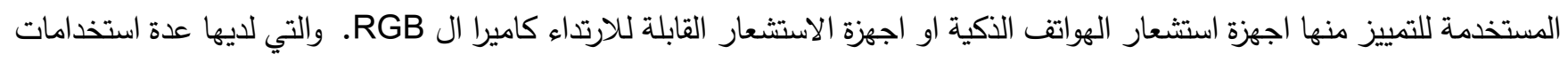
منها تمييز الحركة او قراءة الخصائص الحيوية للجسم او تحديد موقع الشخص, كما تم ذكر الهيكل العام لنظام التعرف على النشاط البشري, علاوة على ذلك, تركز تلك الدراسات على التعرف على الانشطة البشرية وطرق التصنيف المختلفة والمستخدمة في عملية التمييز , تم مقارنة بحوث تتعلق بالانشطة والاجزة المستخدمة في تمييز النشاط البشري ونماذج التعلم وايجيابيات وسلبيات كل خورازمية مستخدمة ومجموعة البيانات ودقة التعلم. واخيرا, تمت مناقثة المميزات والتحديات لكل نوع, وقد اظهرت هذه البحوث بانه قد تم تم استخدام التعلم الالي مؤخرا اكثر من التعلم التقليدي لانه يمتاز بانه اكثر دقة و وتستغرق وقتا اقل عند عملية الاختبار , وقد اظهر ايضا بانه خوارزميه

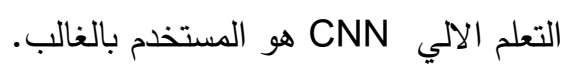




\section{Acknowledgement 8 شكر وتقدير \\ يتقدم الباحث بالثكر و التقدير لكل من يساهم في تقييم البحث وتقديم النصائح والارشادات لاثر اء موضوع الدراسة.}

References : المصادر

[1] Avci,A; Bosch, S.; Marin-Perianu, M.; Marin-Perianu, R.; and Havinga,P. "Activity recognition using inertial sensing for healthcare, wellbeing and sports applications: a survey, "International Conference on Architecture of Computing Systems, 2010.

[2] Oikonomopoulos, A. and Pantic, M. "Human Activity Recognition Using Hierarchically-Mined Feature Constellations," 9th International Symposium, ISVC 2013, Rethymnon, Crete, Greece, July 29-31, 2013. Proceedings, Part I pp. 150-159, 2013.

[3] Javan, R. M. and Levine, M. D. "Human activity recognition in videos using a single example," Image Vis. Comput., vol. 31, no. 11, pp. 864-876, Nov. 2013.

[4] Yang, J.; Lee, J. and Choi, J. "Activity Recognition Based on RFID Object Usage for Smart Mobile Devices,” J. Comput. Sci. Technol., vol. 26, no. 2, pp. 239-246, Mar. 2011.

[5] Chen, L.; Wei, H. and Ferryman, J. "A survey of human motion analysis using depth imagery," Pattern Recognit. Lett., vol. 34, no. 15, pp. 1995-2006, Nov. 2013.

[6] Ong, W.; Palafox, L. and Koseki, T. "Investigation of Feature Extraction for Unsupervised Learning in Human Activity Detection," Bull. Networking, Comput. Syst. Softw., vol. 2, no. 1, pp. 30-35, 2013.

[7] Lara O. D. and Labrador, M. a. "A Survey on Human Activity Recognition using Wearable Sensors," IEEE Commun. Surv. Tutorials, vol. 15, no. 3, pp. 1192-1209, Jan. 2013.

[8] Chaaraoui, A. A.; Padilla-López, J. R.; Climent-Pérez, P. and Flórez-Revuelta, F. "Evolutionary joint selection to improve human action recognition with RGB-D devices," Expert Syst. Appl., vol. 41, no. 3, pp. 786-794, Feb. 2014.

[9] Ryoo, M. S. "Human activity prediction: Early recognition of ongoing activities from streaming videos," in 2011 International Conference on Computer Vision, 2011, no. Iccv, pp. 1036-1043.

[10] Chen, L.; Nugent, C. D. and Wang, H. "A Knowledge-Driven Approach to Activity Recognition in Smart Homes,” IEEE Trans. Knowl. Data Eng., vol. 24, no. 6, pp. 961-974, Jun. 2012.

[11] González-Ortega, D.; Díaz-Pernas, F. J; Martínez-Zarzuela, M. and Antón-Rodríguez, M. "A Kinect-based system for cognitive rehabilitation exercises monitoring.," Comput. Methods Programs Biomed., vol. 113, no. 2, pp. 620-31, Feb. 2014.

[12] Alshurafa, N.; Xu, W.; Liu, J.; Huang, M.-C.; Mortazavi, B.; Roberts, C. and Sarrafzadeh, M. "Designing a Robust Activity Recognition Framework for Health and Exergaming using Wearable Sensors.," IEEE J. Biomed. Heal. informatics, no. c, pp. 1-11, Oct. 2013.

[13] Paragliola, G. and Coronato, A. "Intelligent Monitoring of Stereotyped Motion Disorders in Case of Children with Autism," 2013 9th Int. Conf. Intell. Environ., pp. 258-261, Jul. 2013

[14] Stone, E. and Skubic, M. "Passive, In-Home Gait Measurement Using an Inexpensive Depth Camera: Initial Results," in Proceedings of the 6th International Conference on Pervasive Computing Technologies for Healthcare, 2012, pp. 183-186.

[15] Wang, S.; Zabir, S. and Leibe, B. "Lying Pose Recognition for Elderly Fall Detection," in Robotics:Science and Systems VII, no. 1, H. Durrant-Whyte, N. Roy, and P. Abbeel, Eds. MIT Press, 2012, pp. $345-3$

[16] Luis, J and Ortiz, R. " Smartphone Based Human Activity Recognition," Springer Theses,2014 
[17] Ong, Ch. A.; theng, L. B. "Human Activity Recognition: A Review", 2014 IEEE International Conference on Control System, Computing and Engineering, 28 - 30 November 2014, Penang, Malaysia

[18] Bruno, F. Mastrogiovanni, A. Sgorbissa, T. and Vernazza, R. Z., " Human motion modeling and recognition: a computational approach," IEEE International Conference on Automation Science and Engineering, 2012

[19] Chen, J.; Hoey, C.D.; Nugent, D.J. and Cook, Z. Yu, "Sensor-based activity recognition," IEEE Transactions on Systems, Man, and Cybernetics, Part C: Applications and Reviews 42, 790-808 (2012)

[20] Lara, O.; and Labrador, M. “ A survey on human activity recognition using wearable sensors," IEEE Commun. Surv. Tutorials 1, 1-18 (2012)

[21] Tamas, V. "Human Behavior Recognition In Video Sequences," Technical University of CLUJNAPOCA, 2013

[22] Smisek, J.; Jancosek, M. and Pajdla, T. "3D with Kinect," in 2011 IEEE International Conference on Computer Vision Workshops (ICCV Workshops), 2011, pp. 1154-1160.

[23] Kantoch, E. and Augustyniak, P. "Human activity surveillance based on wearable body sensor network," in Computing in Cardiology (CinC), 2012, pp. 325 - 328.

[24] Slim, S. O.; Atia, A.; Elfattah, M. and Mostafa, M. "Survey on Human Activity Recognition based on Acceleration Data," (IJACSA) International Journal of Advanced Computer Science and Applications, Vol. 10, No. 3, 2019

[25] Arifoglu, D. and Bouchachia, A. "Activity Recognition and Abnormal Behaviour Detection with Recurrent Neural Networks". Procedia Computer Science, 110, pp.86-93, (2017).

[26] Van Kasteren,T; Englebienne, G. and Kr"ose, B. J. A. "Human activity recognition from wireless sensor network data", Benchmark and software. Activity Recognition in Pervasive Intelligent Environments, pages 165-186, 2011.

[27] Hayashi, T.; Nishida, M.; Kitaoka, N.; Toda, T. and Takeda,K. "Daily Activity Recognition with Large-Scaled Real-Life Recording Datasets Based on Deep Neural Network Using Multi-Modal Signals", IEICE Transactions on Fundamentals of Electronics, Communications and Computer Sciences, vol. 101, no. 1, pp. 199-210, 2018

[28] Nhac, L. and Nguyen, T.; Ngo, T.; Nguyen, T. and Nguyen,H. "Mobile Online Activity Recognition System Based on Smartphone Sensors". Advances in Information and Communication Technology: Proceedings of the International Conference, ICTA, pp. 357-366, 2017

[29] Kwapisz, J.R.; Weiss, G.M. and Moore, S.A. "Activity recognition using cell phone accelerometers," ACM SigKDD Explorations Newsletter, vol.12, no.2, pp.74-82, 2011.

[30] Bayat, A.; Pomplun, M. and Tran, D. A. "A study on human activity recognition using accelerometer data from smartphones," Procedia Computer Science, vol. 34, pp. 450-457, 2014.

[31] Alsheikh, M.A.; Selim, A.; Niyato, D.; Doyle, L.; Lin, S. and Tan, H.-P. "Deep Activity Recognition Models with Triaxial Accelerometers," In Proceedings of the AAAIWorkshop: Artificial Intelligence Applied to Assistive Technologies and Smart Environments, Phoenix, AZ, USA, 12 February 2016

[32] Turaga, P. ; Chellappa, R. ; Subrahmanian, V. and Udrea, O. "Machine recognition of human activities: A survey," IEEE Trans. Circuits Syst.Video Technol., vol. 18, no. 11, pp. 1473-1488, 2008.

[33] Xia, L. ; Chen, C. and Aggarwal, J. K. "View invariant human action recognition using histograms of 3D joints," in 2012 IEEE Computer Society Conference on Computer Vision and Pattern Recognition Workshops, 2012, pp. 20-27. 
[34] Vo, Q. V.; Lee, G. and Choi, D. "Fall Detection Based on Movement and Smart Phone Technology," in 2012 IEEE RIVF International Conference on Computing \& Communication Technologies, Research, Innovation, and Vision for the Future, 2012, pp. 1-4

[35] Kaiming, H.; Xiangyu, Zh.; Shaoqing, R., and Jian, S.. "Identity Mappings in Deep Residual Networks,". arXiv 2016.

[36] Ouchi, K. and Doi, M. "Indoor-outdoor Activity Recognition by a Smartphone," In Proceedings of the 2012 ACM Conference on Ubiquitous Computing, Pittsburgh, PA, USA, 5-8 September 2012; pp. 600-601.

[37] Stewart, V.; Ferguson, S.; Peng, J.X. and Rafferty, K. "Practical automated activity recognition using standard smartphones," In Proceeedings of the IEEE International Conference on Pervasive Computing and Communications Workshops, Los Alamitos, CA, USA, 19-23 March 2012; pp. 229-234.

[38] Gomes, J.; Krishnaswamy, S.; Gaber, M.; Sousa, P.; Menasalvas, E. and MARS: A "Personalized Mobile Activity Recognition System," In Proceeding's of the 2012 IEEE 13th International Conference on Mobile Data Management (MDM), Bengaluru, Karnataka, 23-26 July 2012; pp. 316-319.

[39] Lane, N.D.; Mohammod, M.; Lin, M.; Yang, X.; Lu, H.; Ali, S.; Doryab, A.; Berke, E.; Choudhury, T. and Campbell, A. "Bewell: A smartphone application to monitor, model and promote wellbeing," In Proceedings of the 5th International ICST Conference on Pervasive Computing Technologies for Healthcare, Dublin, Ireland, 23-26 May 2011; pp. 23-26

[40] Redmon, J. and Farhadi, A. "Better, faster, stronger. In: Computer Vision and Pattern Recognition," (CVPR), 2017 IEEE Conference on, IEEE (2017) 6517- 6525.

[41] T. Brezmes, J.-luis Gorricho, and J. Cotrina, "Activity Recognition from Accelerometer Data on a Mobile Phone," Test, pp. 796-799, 2009.

[42] L. Sun, D. Zhang, and N. Li, "Physical Activity Monitoring with Mobile Phones," pp. 104-111, 2011. 